\title{
Kripke Semantics for Intersection Formulas
}

\author{
ANDREJ DUDENHEFNER, Saarland University \\ PAWE $Ł$ URZYCZYN, University of Warsaw
}

\begin{abstract}
We propose a notion of the Kripke-style model for intersection logic. Using a game interpretation, we prove soundness and completeness of the proposed semantics. In other words, a formula is provable (a type is inhabited) if and only if it is forced in every model. As a by-product, we obtain another proof of normalization for the Barendregt-Coppo-Dezani intersection type assignment system.
\end{abstract}

CCS Concepts: • Theory of computation $\rightarrow$ Proof theory;

Additional Key Words and Phrases: Intersection types, games in logic, Kripke models

ACM Reference format:

Andrej Dudenhefner and Paweł Urzyczyn. 2021. Kripke Semantics for Intersection Formulas. ACM Trans. Comput. Logic 22, 3, Article 15 (June 2021), 16 pages.

https://doi.org/10.1145/3453481

\section{INTRODUCTION}

Intersection types are broadly used in type assignment systems but are seldom understood as logical formulas. Exceptions include the work of Dezani-Ciancaglini et al. [3], Pottinger [12], Valentini and Viale [19], and Venneri [20], and the line of research on intersection logic initiated by Simona Ronchi Della Rocca and Luca Roversi (e.g., see [11, 13, 14]). Those efforts address mostly proof-theoretical issues, and the underlying semantics of lambda-term assignment is essentially proof theoretical as well. If we think of intersection formulas as independent of term assignment, we naturally ask for mathematical semantics of formulas that could be defined and investigated without any direct reference to lambda terms.

This article proposes the first (to the authors' knowledge) attempt to define a possible-world (Kripke) semantics for intersection logic (the concept being mature enough for a lowercase spelling). This should be distinguished from semantics of conversion and/or type assignment (cf. filter models) where a possible-world approach is also applied. Some works [7, 10, 21] use Kripke frames for purposes quite orthogonal to ours. A more related work is that of Valentini and Viale [19], but this one only addresses the logic of subsumption.

Our approach develops from the idea of a proof search, or type inhabitation algorithm, understood as a game. In an earlier work of the second author [18] such approach was applied to intuitionistic logic, and it seemed natural to be tried on intersection formulas.

Authors' addresses: A. Dudenhefner, Saarland University, Saarland Informatics Campus E1 3, 66123 Saarbrücken, Germany; email: andrej.dudenhefner@cs.uni-saarland.de; P. Urzyczyn, Institute of Informatics, University of Warsaw, Banacha 2, 02097 Warsaw, Poland; email: urzy@mimuw.edu.pl.

This work is licensed under a Creative Commons Attribution International 4.0 License.

(C) 2021 Copyright held by the owner/author(s).

1529-3785/2021/06-ART15 \$15.00

https://doi.org/10.1145/3453481 
The game is played by two competitors. The existential player, ヨros, attempts a top-down construction of a normal inhabitant (proof term). His impatience is tempered by the universal reviewer, $\forall$ phrodite. The game admits determinacy derived from De Morgan's laws. Therefore, we either have a proof (winning $\exists$ ros' strategy) or a refutation (winning $\forall$ phrodite's strategy). From the former, we obtain normalization-that is, existence of normal proofs. From the latter, a Kripke countermodel can be constructed. This constitutes a completeness proof.

The adaptation of the preceding to intersection logic makes the picture a bit more obscure for two reasons. One is that in the proof construction process, one must necessarily consider parallel inhabitation problems, where a single proof is expected to satisfy multiple constraints (cf. [9, 16, 17]). Such parallel problems are naturally represented as matrices of types: each row represents a single constraint, whereas columns correspond to variables. This results in complex syntax (we define a sequent calculus using matrices of formulas) and an intricate model definition.

The other reason, quite cumbersome, is that intersection formulas may be non-uniform, such as the formula $p \cap(q \rightarrow p)$, exhibiting "functional" and "atomic" behavior. To accommodate both, our models must satisfy a global condition of being "monotone." Although this restriction may appear artificial, it seems unavoidable. (Note that such global constraints on models are not unusual, cf. models for second-order intuitionistic logic $[5,6,15]$.) On the positive side, let us note that the monotonicity condition is trivially satisfied if only refinements of simple types [8] are considered.

In what follows, we first define the syntax of matrices and columns and introduce a sequent calculus system to derive columns from matrices. We show that our sequent calculus is equivalent to the Barendregt-Coppo-Dezani (BCD) intersection type assignment $[1,2]$. Then we define Kripke-style models and intersection games, and generalize the main results of Urzyczyn [18] in the new setting. We show soundness and completeness of our semantics (Theorem 36) and proof normalization (Theorem 39).

\section{FORMULAS AND MATRICES}

Formulas, ranged over by $\alpha, \beta, \sigma, \tau, \rho, \varepsilon$, are $\mathrm{BCD}$ intersection types [1]-that is,

$$
\sigma, \tau::=p|\omega| \sigma \rightarrow \tau \mid \sigma \cap \tau,
$$

where atoms are ranged over by $p, q, r, s$. The symbol $\leq$ denotes intersection type subtyping (i.e., the least preorder on types satisfying the following conditions):

$$
\begin{gathered}
\sigma \leq \omega, \quad \omega \leq \omega \rightarrow \omega, \quad \sigma \cap \tau \leq \sigma, \quad \sigma \cap \tau \leq \tau, \quad \sigma \leq \sigma \cap \sigma, \\
\quad(\sigma \rightarrow \tau) \cap(\sigma \rightarrow \rho) \leq \sigma \rightarrow \tau \cap \rho ;
\end{gathered}
$$

The intersection type constructor $(\cap)$ is assumed commutative, associative, and idempotent. The universal type $\omega$ is identified with the empty intersection, and we also identify $\sigma \cap \omega$ with $\sigma$.

If $\sigma=\tau \cap \rho$, then we may write $\tau \subseteq \sigma$ (in this case, we also have $\sigma \leq \tau$ ). A formula $\sigma$ is functional if $\sigma=\bigcap_{i \in I}\left(\alpha_{i} \rightarrow \beta_{i}\right)$. In particular, the formula $\omega$ is functional. A non-functional formula is always of the form $\sigma=\bigcap_{i \in I} \tau_{i}$, where at least one $\tau_{i}$ is an atom.

A vector of formulas of length $m$ is called a column of height $m$. The width of a column is the number of arrows in its coordinate for which this number is maximal. A column is called functional when all of its coordinates are functional; otherwise, it is atomic. Columns are ranged over by $\gamma, \delta, \zeta, v$. The column $(\omega, \ldots, \omega)$ is denoted by $\omega$. (Columns should in principle be vertical, but we sometimes represent them as horizontal vectors to save space.)

An $m \times n$-matrix of formulas $\sigma_{i j}$, where $i=1 \ldots m$ and $j=1 \ldots n$, is written $\left[\sigma_{i j}\right]_{j=1 \ldots n}^{i=1 \ldots m}$, but this notation may be abbreviated in various ways. Such a matrix has $m$ rows of the form $\left(\sigma_{i 1}, \ldots, \sigma_{i n}\right)$ and $n$ columns of the form $\left(\sigma_{1 j}, \ldots, \sigma_{m j}\right)$. We assume $m \geq 1$ and $n \geq 0$-that is, a matrix must 
have at least one, possibly empty, row. Matrices are ranged over by $\Gamma$. If $\Gamma$ is an $m \times n$-matrix, and $\gamma$ is a column of height $m$, then $\Gamma, \gamma$ stands for an $m \times(n+1)$-matrix obtained by adding $\gamma$ as the $(n+1)$-st column.

A matrix is atomic when all of its columns are atomic. The relation $\leq$ extends to columns coordinatewise. If $\gamma \leq \gamma^{\prime}$, for some column $\gamma$ of $\Gamma$, then we may write $\Gamma \leq \gamma^{\prime}$ for simplicity.

If $\gamma=\left(\sigma_{1}, \ldots, \sigma_{m}\right)$ is a column and $\tau_{i} \subseteq \sigma_{i}$, for $i=1 \ldots m$, then the column $\delta=\left(\tau_{1}, \ldots, \tau_{m}\right)$ is a subcolumn of $\gamma$, written $\delta \subseteq \gamma$. A subcolumn $\delta$ of a matrix $\Gamma$ is a subcolumn of one of its columns, written $\delta \subseteq \Gamma$.

Example 1. Let $\Gamma=\left(\begin{array}{cc}p \cap(p \rightarrow q) & q \\ q \rightarrow p & \omega\end{array}\right)$ be a $2 \times 2$-matrix. Each column in $\Gamma$ contains at least one atomic coordinate. Therefore, $\Gamma$ is atomic. All functional subcolumns of $\Gamma$ are $\left(\begin{array}{c}\omega \\ \omega\end{array}\right),\left(\begin{array}{c}p \rightarrow q \\ \omega\end{array}\right),\left(\begin{array}{c}\omega \\ q \rightarrow p\end{array}\right)$, and $\left(\begin{array}{c}p \rightarrow q \\ q \rightarrow p\end{array}\right)$.

Let $f:\left\{1, \ldots, m_{2}\right\} \rightarrow\left\{1, \ldots, m_{1}\right\}$ be onto. If $\gamma=\left(\sigma_{1}, \ldots, \sigma_{m_{2}}\right)$, then $f(\gamma)$ is a column of height $m_{1}$ whose $k$-th coordinate is $\bigcap_{f(i)=k} \sigma_{i}$. And if $\delta=\left(\tau_{1}, \ldots, \tau_{m_{1}}\right)$, then $f^{-1}(\delta)$ is a column of height $m_{2}$ whose $j$-th coordinate is $\tau_{f(j)}$. Observe that $f\left(f^{-1}(\delta)\right)=\delta$, up to idempotency, whereas only $\gamma \subseteq f^{-1}(f(\gamma))$ holds in general.

Example 2. Consider $f:\{1,2\} \rightarrow\{1\}$ such that $f(1)=f(2)=1$. Then

$$
f^{-1}\left(f\left(\left(\begin{array}{l}
p \\
q
\end{array}\right)\right)\right)=f^{-1}((p \cap q))=\left(\begin{array}{c}
p \cap q \\
p \cap q
\end{array}\right) \text { and } f\left(f^{-1}((p))\right)=f\left(\left(\begin{array}{l}
p \\
p
\end{array}\right)\right)=(p \cap p)=(p) \text {. }
$$

The notation $f^{-1}$ extends to matrices columnwise: if $\Gamma$ is an $m_{1} \times n$-matrix, then $f^{-1}(\Gamma)$ is an $m_{2} \times n$-matrix whose $i$-th row is the $f(i)$-th row of $\Gamma$. In other words, $f^{-1}(\Gamma)$ is obtained from $\Gamma$ by copying its rows (each at least once).

The relation $\sqsubseteq$ on matrices represents a matrix expansion by copying rows and adding new columns. In other words, we write $\Gamma_{1} \sqsubseteq_{f} \Gamma_{2}$ if there exist columns $\gamma_{1}, \ldots, \gamma_{k}$ such that $\Gamma_{2}=$ $f^{-1}\left(\Gamma_{1}\right), \gamma_{1}, \ldots, \gamma_{k}$.

FACT 3. If $\Gamma_{1} \sqsubseteq_{f} \Gamma_{2}$ and $\Gamma_{2} \sqsubseteq_{g} \Gamma_{3}$, then $\Gamma_{1} \sqsubseteq_{f \circ g} \Gamma_{3}$.

Example 4. Let $\Gamma_{1}=\left(\begin{array}{l}p \\ q\end{array}\right)$ be a $2 \times 1$-matrix, and let $\Gamma_{2}=\left(\begin{array}{ll}p & r \\ q & r \\ p & p\end{array}\right)$ be a $3 \times 2$-matrix. Then $\Gamma_{1} \sqsubseteq_{f} \Gamma_{2}$, where $f:\{1,2,3\} \rightarrow\{1,2\}$, and $f(1)=f(3)=1$ and $f(2)=2$.

If $\gamma=\left(\sigma_{1}, \ldots, \sigma_{m}\right)$ and $\delta=\left(\tau_{1}, \ldots, \tau_{m}\right)$, then the column $\left(\sigma_{1} \rightarrow \tau_{1}, \ldots, \sigma_{m} \rightarrow \tau_{m}\right)$ is denoted by $\gamma \Rightarrow \delta$. If $\gamma=\left(\bigcap_{i \in I_{1}}\left(\alpha_{1 i} \rightarrow \beta_{1 i}\right), \ldots, \bigcap_{i \in I_{m}}\left(\alpha_{m i} \rightarrow \beta_{m i}\right)\right)$ is a functional column, then we define $\operatorname{lhs}(\gamma)=\left(\bigcap_{i \in I_{1}} \alpha_{1 i}, \ldots, \bigcap_{i \in I_{m}} \alpha_{m i}\right)$ and $\operatorname{rhs}(\gamma)=\left(\bigcap_{i \in I_{1}} \beta_{1 i}, \ldots, \bigcap_{i \in I_{m}} \beta_{m i}\right)$.

Observe that if $\gamma \neq \omega$, then the width of $\operatorname{lhs}(\gamma)$ and $\operatorname{rhs}(\gamma)$ is less than the width of $\gamma$. We remark that $\operatorname{lhs}(\delta \Rightarrow \gamma)=\delta \operatorname{rhs}(\delta \Rightarrow \gamma)=\gamma$, and $\operatorname{lhs}(\omega)=\operatorname{rhs}(\omega)=\omega$. However, $\operatorname{lhs}(\delta) \Rightarrow \operatorname{rhs}(\delta)$ is typically not the same as $\delta$.

LEMma 5. If $\gamma \leq \gamma^{\prime}$ and $v^{\prime}$ is a functional subcolumn of $\gamma^{\prime}$, then there is a functional subcolumn $v$ of $\gamma$ such that $\operatorname{lhs}\left(v^{\prime}\right) \leq \operatorname{lhs}(v)$ and $\operatorname{rhs}(v) \leq \operatorname{rhs}\left(v^{\prime}\right)$.

Proof. Let $v^{\prime}=\left(\tau_{1}, \ldots, \tau_{m}\right)$ where $\tau_{k}=\bigcap\left\{\alpha_{k i} \rightarrow \beta_{k i} \mid i \in I_{k}\right\}$, and let $\gamma=\left(\sigma_{1}, \ldots, \sigma_{m}\right)$ with $\sigma_{k}=\bigcap\left\{\rho_{k j} \rightarrow \varepsilon_{k j} \mid j \in J_{k}\right\} \cap \bigcap\left\{p_{\ell} \mid \ell \in L_{k}\right\}$, where $p_{\ell}$ are atoms. We have $\gamma^{\prime} \leq v^{\prime}$, whence also $\gamma \leq v^{\prime}$ (i.e., $\sigma_{k} \leq \tau_{k}$ ) for $k=1 \ldots m$.

Consider any $k=1 \ldots m$ such that $I_{k} \neq \varnothing$ (i.e., $\tau_{k} \neq \omega$ ). Then by beta soundness [2, Def. 14.1.4, Thm. 14.1.7], there are non-empty sets $L_{k}^{i} \subseteq J_{k}$ with $\alpha_{k i} \leq \bigcap\left\{\rho_{k j} \mid j \in L_{k}^{i}\right\}$ and $\bigcap\left\{\varepsilon_{k j} \mid j \in L_{k}^{i}\right\} \leq \beta_{k i}$, for $i \in I_{k}$. Define a type $\sigma_{k}^{\prime}=\bigcap\left\{\rho_{k j} \rightarrow \varepsilon_{k j} \mid j \in \bigcup\left\{L_{k}^{i} \mid i \in I_{k}\right\}\right\}$.

In case $I_{k}=\varnothing$, we take $\sigma_{k}^{\prime}=\omega$. Then $v=\left(\sigma_{1}^{\prime}, \ldots, \sigma_{m}^{\prime}\right)$ is a subcolumn of $\gamma$ satisfying the conditions $\operatorname{lhs}\left(v^{\prime}\right) \leq \operatorname{lhs}(v)$ and $\operatorname{rhs}(v) \leq \operatorname{rhs}\left(v^{\prime}\right)$. 


$$
\begin{aligned}
& \frac{(\Gamma \leq \gamma)}{\Gamma \vdash \gamma}(\mathrm{A}) \quad \frac{\Gamma \vdash \gamma \quad \Gamma, \gamma \vdash \delta}{\Gamma \vdash \omega}(\Omega) \quad \frac{\Gamma \mathrm{Cut})}{\Gamma \vdash \delta} \\
& \frac{\Gamma, \operatorname{rhs}(\gamma) \vdash \delta \quad \Gamma \vdash \operatorname{lhs}(\gamma) \quad(\gamma \subseteq \Gamma)}{\Gamma \vdash \delta}(\mathrm{L}) \quad \frac{f^{-1}(\Gamma), \gamma \vdash \delta}{\Gamma \vdash f(\gamma \Rightarrow \delta)}(\mathrm{R})
\end{aligned}
$$

Fig. 1. Sequent calculus.

Lemma 6. If $\delta \leq f(\gamma \Rightarrow \zeta)$, then there exists a functional subcolumn $v$ of $f^{-1}(\delta)$ such that $\gamma \leq \operatorname{lhs}(v)$ and $\operatorname{rhs}(v) \leq \zeta$.

Proof. The inequality $\delta \leq f(\gamma \Rightarrow \zeta)$ implies $f^{-1}(\delta) \leq f^{-1}(f(\gamma \Rightarrow \zeta))$. The column $\gamma \Rightarrow \zeta$ is a functional subcolumn of $f^{-1}(f(\gamma \Rightarrow \zeta))$. Therefore, the sought subcolumn of $f^{-1}(\delta)$ exists by Lemma 5.

\section{SEQUENT CALCULUS}

fudgments (compare to the molecules of Ronchi Della Rocca [14]) take the form $\Gamma \vdash \gamma$, where $\Gamma$ is a matrix and $\gamma$ is a column of the same height. Rules of our sequent calculus are given in Figure 1. Observe that we only have two logical rules, the left rule $(\mathrm{L})$ and the right rule $(\mathrm{R})$, rather than separate rules for the type constructors $(\rightarrow)$ and $(\cap)$. In rule $(\mathrm{L})$, the column $\gamma$ must be functional. Structural rules and subtyping rules are admissible (Lemmas 7 and 8).

Lemma 7 (Structural Rules).

(1) If $\Gamma \vdash \delta$ and $\Gamma^{\prime}$ is a matrix obtained by permuting columns of $\Gamma$, then $\Gamma^{\prime} \vdash \delta$.

(2) If $\Gamma \vdash \delta$, then $\Gamma, \gamma \vdash \delta$.

(3) If $\Gamma, \gamma, \gamma \vdash \delta$, then $\Gamma, \gamma \vdash \delta$.

Proof. (1) Structural induction with respect to proofs, showing a stronger induction hypothesis that proof size (number of rule applications) does not increase.

(2), (3) Induction with respect to proof size using (1).

LEMMA 8 (SUbTyping Rules).

(1) If $\Gamma, \gamma^{\prime} \vdash \delta$ and $\gamma \leq \gamma^{\prime}$, then $\Gamma, \gamma \vdash \delta$.

(2) If $\Gamma \vdash \delta$ and $\delta \leq \delta^{\prime}$, then $\Gamma \vdash \delta^{\prime}$.

Proof. (1) By rule (A), we have $\Gamma, \gamma \vdash \gamma^{\prime}$. By Lemma 7(2) and Lemma 7(1), we have $\Gamma, \gamma, \gamma^{\prime} \vdash \delta$. The claim follows by rule (Cut).

(2) By rule (A), we have $\Gamma, \delta \vdash \delta^{\prime}$. The claim follows by rule (Cut).

We need to show that our sequent calculus is sound and complete with respect to the BCD type assignment system (Figure 2). For this, we generalize BCD judgments as follows. If $\Gamma=\left[\sigma_{i j}\right]_{j=1 \ldots n}^{i=1 \ldots m}$ and $\delta=\left(\tau_{1}, \ldots, \tau_{m}\right)$, then $\Gamma \vdash_{\mathrm{BCD}} M: \delta$ means that, in the BCD system,

$$
\left\{x_{1}: \sigma_{i 1}, \ldots, x_{n}: \sigma_{i n}\right\} \vdash M: \tau_{i} \text {, for } i=1 \ldots m .
$$

In such circumstances, if $\gamma_{j}$ is the $j$-th column of $\Gamma$, then we write $x_{j}: \gamma_{j}$. Notation $\Gamma \vdash_{\text {BCD }} \gamma$ means that $\Gamma \vdash_{\mathrm{BCD}} M: \gamma$, for some term $M$. If $M$ is in $\beta$-normal form, then we may write $\Gamma \vdash_{\mathrm{BCD}}^{\mathrm{N}} \gamma$. 


$$
\begin{array}{cc}
\overline{\Gamma, x: \sigma \vdash x: \sigma} & \overline{\Gamma \vdash M: \omega} \\
\frac{\Gamma, x: \sigma \vdash M: \tau}{\Gamma \vdash \lambda x: M: \sigma \rightarrow \tau} & \frac{\Gamma \vdash M: \sigma \rightarrow \tau \quad \Gamma \vdash N: \sigma}{\Gamma \vdash M N: \tau} \\
\frac{\Gamma \vdash M: \sigma \quad \Gamma \vdash M: \tau}{\Gamma \vdash M: \sigma \cap \tau} & \frac{\Gamma \vdash M: \sigma}{\Gamma \vdash M: \tau}(\sigma \leq \tau)
\end{array}
$$

Fig. 2. BCD type assignment.

Example 9. Let $\Gamma=\left(\begin{array}{c}p \cap(p \rightarrow q) \\ \omega \rightarrow r\end{array}\right)$ be a $2 \times 1$-matrix. First, we have $\Gamma \vdash_{\mathrm{BCD}}\left(\begin{array}{c}q \\ r\end{array}\right)$, because $\left\{x_{1}: p \cap(p \rightarrow q)\right\} \vdash_{\mathrm{BCD}} x_{1} x_{1}: q$ and $\left\{x_{1}: \omega \rightarrow r\right\} \vdash_{\mathrm{BCD}} x_{1} x_{1}: r$. Since $x_{1} x_{1}$ is in $\beta$-normal form, we have $\Gamma \vdash_{\mathrm{BCD}}^{\mathrm{N}}\left(\begin{array}{c}q \\ r\end{array}\right)$. Second, we have $\Gamma \vdash\left(\begin{array}{c}q \\ r\end{array}\right)$ by the following derivation:

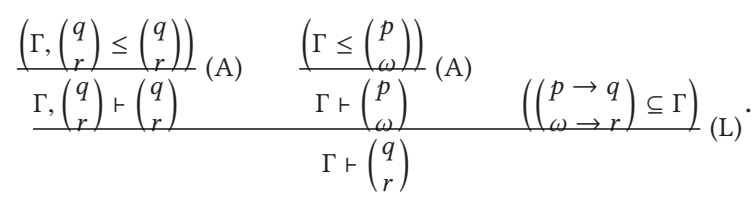

Observe that the preceding derivation does not use the cut rule.

The following is an immediate consequence of Barendregt et al. [2, Prop. 13.2.10].

LEMMA 10. If $\Gamma, x: \delta \vdash_{\mathrm{BCD}} M: \gamma$, and $\Gamma \vdash_{\mathrm{BCD}} N: \delta$, then $\Gamma \vdash_{\mathrm{BCD}} M[x:=N]: \gamma$.

Our sequent calculus is sound and complete with respect to BCD inhabitation.

Lemma 11. The conditions $\Gamma \vdash \gamma$ and $\Gamma \vdash_{\mathrm{BCD}} \gamma$ are equivalent. In addition, if $\Gamma \vdash \gamma$ has a cut-free proof, then $\Gamma \vdash_{\mathrm{BCD}}^{\mathrm{N}} \gamma$.

Proof. (Soundness) The proof is by induction with respect to proofs. For the axiom (A), we have (slightly abusing the notation) that $\Gamma \vdash_{\mathrm{BCD}} x: \gamma$, where some $x: \gamma^{\prime}$ is in $\Gamma$ and $\gamma^{\prime} \leq \gamma$. Rule $(\Omega)$ is obviously sound. For rule (Cut), assume $\Gamma \vdash_{\text {всD }} M: \gamma$ and $\Gamma, x: \gamma \vdash_{\text {ВсD }} N: \delta$. Then $\Gamma \vdash_{\mathrm{BCD}} N[x:=M]: \delta$ holds by Lemma 10 .

For rule (L), let $\gamma=\left(\bigcap_{j \in I_{1}}\left(\alpha_{1 j} \rightarrow \beta_{1 j}\right), \ldots, \bigcap_{j \in I_{m}}\left(\alpha_{m j} \rightarrow \beta_{m j}\right)\right)$ and $\delta=\left(\tau_{1}, \ldots, \tau_{m}\right)$.

Assume $\Gamma, x: \operatorname{rhs}(\gamma) \vdash_{\mathrm{BCD}} M: \delta$ and $\Gamma \vdash_{\mathrm{BCD}} N: \operatorname{lhs}(\gamma)$. Then $\Gamma_{i}, x: \bigcap_{j \in I_{i}} \beta_{i j} \vdash_{\mathrm{BCD}} M: \tau_{i}$ and $\Gamma_{i} \vdash_{\mathrm{BCD}} N: \bigcap_{j \in I_{i}} \alpha_{i j}$, where $\Gamma_{i}$ corresponds to the $i$-th row of $\Gamma$. Then by Lemma 10, we have $\Gamma_{i}, y: \bigcap_{j \in I_{i}}\left(\alpha_{i j} \rightarrow \beta_{i j}\right) \vdash_{\mathrm{BCD}} M[x:=y N]: \tau_{i}$, showing $\Gamma, y: \gamma \vdash_{\mathrm{BCD}} M[x:=y N]: \delta$. Therefore, we have $\Gamma \vdash_{\mathrm{BCD}} \delta$, because $\Gamma \leq \gamma$. In addition, if terms $M$ and $N$ are normal, then so is $M[x:=y N]$.

Finally, let us consider rule (R). Let $\Gamma^{\prime}=f^{-1}(\Gamma)$, and assume that $\Gamma^{\prime}, x: \gamma \vdash_{\text {BCD }} M: \delta$ where $\gamma=\left(\sigma_{1}, \ldots, \sigma_{m_{2}}\right)$ and $\delta=\left(\tau_{1}, \ldots, \tau_{m_{2}}\right)$. By the assumption, $\Gamma_{i}^{\prime}, x: \sigma_{i} \vdash_{\text {всD }} M: \tau_{i}$, for $i=1 \ldots m_{2}$, where $\Gamma_{i}^{\prime}$ represents the $i$-th row of $\Gamma^{\prime}$ (which is the same as the $f(i)$-th row of $\Gamma$ ). Then $\Gamma_{i}^{\prime} \vdash_{\mathrm{BCD}} \lambda x . M: \sigma_{i} \rightarrow \tau_{i}$, for $i=1 \ldots m_{2}$. This implies $\Gamma \vdash_{\mathrm{BCD}} \lambda x . M: f(\gamma \Rightarrow \delta)$, showing the claim.

(Completeness) The proof of completeness is by induction with respect to the term $M$ witnessing $\Gamma \vdash_{\mathrm{BCD}} \gamma$. To fix the notation, assume that $\Gamma=\left[\sigma_{i j}\right]_{j=1 \ldots n}^{i=1 \ldots m}$ and $\gamma=\left(\tau_{1}, \ldots, \tau_{m}\right)$, and that variables $x_{1}, \ldots, x_{n}$ correspond to the $n$ columns of $\Gamma$. If $M=x_{j}$, for some $j \in\{1 \ldots n\}$, then we have $\sigma_{i j} \leq \tau_{i}$, for $i=1 \ldots m$, by the generation lemma [2, Thm. 14.1.9]. Thus, $\Gamma \vdash \gamma$ by the axiom (A). If $M$ is a variable other than $x_{j}$, then the only option is $\gamma=\omega$, so the judgment follows by rule $(\Omega)$. 
Assume that $M=P Q$. The generation lemma yields that, for some types $\rho_{i}$,

$$
\left\{x_{1}: \sigma_{i 1}, \ldots, x_{n}: \sigma_{i n}\right\} \vdash_{\mathrm{BCD}} P: \rho_{i} \rightarrow \tau_{i} \text { and }\left\{x_{1}: \sigma_{i 1}, \ldots, x_{n}: \sigma_{i n}\right\} \vdash_{\mathrm{BCD}} Q: \rho_{i} \text {. }
$$

Let $\delta=\left(\rho_{1}, \ldots, \rho_{m}\right)$. By the induction hypothesis, we have $\Gamma \vdash \delta \Rightarrow \gamma$ and $\Gamma \vdash \delta$. Hence, also $\Gamma, \delta \Rightarrow \gamma \vdash \delta$, by Lemma $7(2)$.

From $\Gamma, \delta \Rightarrow \gamma, \gamma \vdash \gamma$ and $\Gamma, \delta \Rightarrow \gamma \vdash \delta$, we derive $\Gamma, \delta \Rightarrow \gamma \vdash \gamma$ by rule (L) using the functional column $\delta \Rightarrow \gamma$. From $\Gamma \vdash \delta \Rightarrow \gamma$, we obtain $\Gamma \vdash \gamma$ by rule (Cut).

If $M=\lambda x$. $N$, then, by the generation lemma, the column $\gamma$ must be a functional column-that is, $\tau_{i}=\bigcap_{k \in I_{i}}\left(\alpha_{k}^{i} \rightarrow \beta_{k}^{i}\right)$, for $i=1 \ldots m$. In addition, $\left\{x_{1}: \sigma_{i 1}, \ldots, x_{n}: \sigma_{i n}, x: \alpha_{k}^{i}\right\} \vdash_{\mathrm{BCD}} N: \beta_{k}^{i}$, for $i=$ $1 \ldots m$ and $k \in I_{i}$. Assuming that every set $I_{i}$ consists of $e_{i}$ elements, we now have $e=e_{1}+\cdots+e_{m}$ assertions about $N$. To them, we apply induction to conclude that $\Gamma^{\prime}, \zeta \vdash \delta$, where

- $\Gamma^{\prime}=f^{-1}(\Gamma)$, for an appropriate surjection $f:\{1, \ldots, e\} \rightarrow\{1, \ldots, m\}$;

- Columns $\zeta$ and $\delta$ consist of all $\alpha_{k}^{i}$ and all $\beta_{i}^{k}$, respectively, in the order consistent with $f$ (i.e., such that $\gamma=f(\zeta \Rightarrow \delta)$ ).

Therefore, we obtain $\Gamma \vdash \gamma$ by rule (R).

\section{KRIPKE-STYLE SEMANTICS}

Our semantics is an adaptation of the standard pattern used in intuitionistic logic [4] to the needs of intersection logic. We define a Kripke model as $\mathcal{M}=\langle C, \leq, \mathcal{G}, \mathcal{H}\rangle$, where

- $C$ is a non-empty set of states;

- $\leq$ is a partial order on $C$;

- $\mathcal{G}$ is a function that assigns an atomic matrix $\Gamma^{C}$ to every state $C \in C$;

- $\mathcal{H}$ is a function that assigns to every pair $C \leq D$ a surjection $f$ satisfying $\Gamma^{C} \sqsubseteq_{f} \Gamma^{D}$ (this is written as $\left.C \leq_{f} D\right)$, in such a way that $\mathcal{H}(C \leq E)=\mathcal{H}(C \leq D) \circ \mathcal{H}(D \leq E)$, whenever $C \leq D \leq E$-in other words, if $C \leq_{f} D \leq_{g} E$, then $C \leq_{f \circ g} E$.

Lemma 12. For every state $C$, we have $\mathcal{H}(C \leq C)=\mathrm{id}$.

Proof. If $C \leq_{f} C$, then $f=f^{2}$, since $C \leq C \leq C$. The only such surjection is identity.

Forcing: Let $\Gamma^{C}$ and $\delta$ be of height $m$. The state $C$ forces $\delta$, written $C \Vdash \delta$, when either $\delta$ is equivalent to $\omega$ under subtyping, or one of the following holds:

- The column $\delta$ is atomic, and $\Gamma^{C} \leq \delta$.

- The column $\delta$ is functional, and

for all $D \in C$ such that $C \leq_{f} D$, and for every subcolumn $v$ of $f^{-1}(\delta)$,

if $D \Vdash \operatorname{lhs}(v)$, then $D \Vdash \operatorname{rhs}(v)$.

Our Kripke models satisfy the following monotonicity property.

Lemma 13 (Monotonicity I). If $C \Vdash \delta$ and $C \leq_{f} D$, then $D \Vdash f^{-1}(\delta)$.

Proof. If $\delta$ is atomic, then so is $f^{-1}(\delta)$, and the claim follows due to $\Gamma^{C} \sqsubseteq_{f} \Gamma^{D}$. So assume that $\delta$ is functional, and let $D \leq_{g} E$. Consider a subcolumn $v$ of $g^{-1}\left(f^{-1}(\delta)\right)$ such that $E \Vdash l h s(v)$. We have $E \Vdash \operatorname{rhs}(v)$, because $C \leq_{f \circ g} E$, and $v$ is a subcolumn of $(f \circ g)^{-1}(\delta)$.

Unfortunately, the semantics based on arbitrary models is not sound.

Example 14. Let $\sigma=(p \cap(p \rightarrow q)) \rightarrow q$. Consider a one-state model with $C=\{1\}$, where $\Gamma^{1}=(p \cap(p \rightarrow q))$ is a $1 \times 1$-matrix. We have $\varnothing \vdash_{\mathrm{BCD}} \lambda x . x x: \sigma$. However, $1 \nVdash(\sigma)$, because $1 \Vdash(p \cap(p \rightarrow q)), 1 \preceq_{\text {id }} 1$, and $1 \nVdash(q)$.

This is because atomic columns can have functional subcolumns, and those are expected to exhibit functional behavior, not guaranteed by their mere occurrence in $\Gamma^{C}$. Therefore, we must restrict attention to a subclass of models. 
We say that a model is monotone if it satisfies the following condition:

For every $C$ and $\gamma$, if $\gamma$ is a subcolumn of $\Gamma^{C}$, then $C \Vdash \gamma$.

The model in Example 14 is not monotone, because $(p \rightarrow q)$ is a subcolumn of $\Gamma^{\mathbf{1}}$, and $\mathbf{1} \nVdash p \rightarrow q$. Note however that a model where all $\Gamma^{C}$ consist only of atoms is trivially monotone. From now on, we only consider monotone models.

Let us illustrate forcing in monotone models. In Example 15 and Example 16, we focus our attention on models with matrices of height 1 . Such models resemble ordinary Kripke models: just identify single formulas with one-row columns. For instance, the following is the standard countermodel for Peirce's law.

Example 15. Let $\delta=(((p \rightarrow q) \rightarrow p) \rightarrow p)$ be a column of height 1 and $C=\{1,2\}$, where $\Gamma^{1}=()$ is a $1 \times 0$-matrix (i.e., a matrix of one empty row), $\Gamma^{2}=(p)$ is a $1 \times 1$-matrix, and $1 \unlhd_{\mathrm{id}} 2$. We have that $1 \nVdash(p \rightarrow q)$, because $1 \preceq_{\mathrm{id}} 2,(p \rightarrow q) \subseteq \mathrm{id}^{-1}(p \rightarrow q), 2 \Vdash(p)$, and $2 \nVdash(q)$. Additionally, $2 \nVdash(p \rightarrow q)$. Therefore, we have that $\mathbf{1} \Vdash((p \rightarrow q) \rightarrow p)$. Finally, we obtain $\mathbf{1} \nVdash \delta$, because $\mathbf{1} \preceq_{\text {id }} \mathbf{1}$, $\delta \subseteq \operatorname{id}^{-1}(\delta), 1 \Vdash \operatorname{lhs}(\delta)=((p \rightarrow q) \rightarrow p)$, and $1 \nVdash \operatorname{rhs}(\delta)=(p)$. Overall, the described model is monotone and contains a state that does not force $\delta$.

Example 16. Let $\delta=((p \rightarrow \omega \rightarrow p) \cap(\omega \rightarrow p \rightarrow p))$ be a column of height 1 and $C=\{\mathbf{1}\}$, where $\Gamma^{1}=(p)$ is a $1 \times 1$-matrix. We have that $\delta$ is functional, $\mathbf{1} \preceq_{\mathrm{id}} \mathbf{1}$, and there are four possible subcolumns of $\operatorname{id}^{-1}(\delta)$, namely $v_{1}=(\omega), v_{2}=(p \rightarrow \omega \rightarrow p), v_{3}=(\omega \rightarrow p \rightarrow p)$, and $v_{4}=\delta$. Then

$1 \Vdash \operatorname{lhs}\left(v_{1}\right)=\operatorname{rhs}\left(v_{1}\right)=(\omega)$.

$1 \Vdash$ lhs $\left(v_{2}\right)=(p)$ and $1 \Vdash \operatorname{rhs}\left(v_{2}\right)=(\omega \rightarrow p)$, because $1 \Vdash(p)$.

$1 \Vdash \operatorname{lhs}\left(v_{3}\right)=(\omega)$ and $1 \Vdash \operatorname{rhs}\left(v_{3}\right)=(p \rightarrow p)$, because $1 \Vdash(p)$.

$1 \Vdash \operatorname{lhs}\left(v_{4}\right)=(p \cap \omega)=(p)$ and $1 \Vdash \operatorname{rhs}\left(v_{4}\right)=((\omega \rightarrow p) \cap(p \rightarrow p))$, because ultimately $1 \Vdash(p \cap p)=(p)$. Overall, we have that $1 \Vdash \delta$.

Interestingly, $\delta=((p \rightarrow \omega \rightarrow p) \cap(\omega \rightarrow p \rightarrow p))$ is forced in every state in a monotone model with matrices of height 1 . Essentially, there are only three such states that are non-equivalent: the column $(p)$ is forced now, later, or never. However, the only normal inhabitant of the formula $p \rightarrow \omega \rightarrow p$ is $\lambda x . \lambda y . x$ and the only normal inhabitant of the formula $\omega \rightarrow p \rightarrow p$ is $\lambda x . \lambda y . y$. Therefore, there is no normal form $M$ such that $\vdash_{\mathrm{BCD}}^{\mathrm{N}} M: \delta$. To construct a countermodel for $\delta$, we have to consider models with matrices of greater height.

Example 17. Let $\delta$ be as in Example 16, and consider the model $C=\{\mathbf{1}, 2\}$, where $\Gamma^{\mathbf{1}}=()$ is a $1 \times 0$-matrix, $\Gamma^{2}=\left(\begin{array}{cc}p & \omega \\ \omega & p\end{array}\right)$ is a $2 \times 2$-matrix, and $1 \unlhd_{f} 2$, where $f:\{1,2\} \rightarrow\{1\}$ is such that $f(1)=f(2)=1$. For $v=\left(\begin{array}{c}p \rightarrow \omega \rightarrow p \\ \omega \rightarrow p \rightarrow p \rightarrow p\end{array}\right)$, we have that $1 \leq_{f} 2, v \subseteq f^{-1}(\delta)$, and $2 \Vdash \operatorname{lhs}(v)=\left(\begin{array}{c}p \\ \omega\end{array}\right)$. However, $2 \nVdash \operatorname{rhs}(v)=\left(\begin{array}{c}\omega \rightarrow p \\ p \rightarrow p\end{array}\right)$, because $2 \Vdash \operatorname{lhs}(\operatorname{rhs}(v))=\left(\begin{array}{c}\omega \\ p\end{array}\right)$, but $2 \nVdash \operatorname{rhs}(\operatorname{rhs}(v))=\left(\begin{array}{c}p \\ p\end{array}\right)$. Overall, we have that $1 \nVdash \delta$, providing a countermodel.

Example 18. Let $\sigma=\sigma_{1} \cap \sigma_{2}$, where $\sigma_{1}=(p \rightarrow(r \rightarrow q) \rightarrow r \rightarrow(p \rightarrow q) \rightarrow q)$ and $\sigma_{2}=(p \rightarrow(p \rightarrow q) \rightarrow r \rightarrow(r \rightarrow q) \rightarrow q)$. A countermodel for $\sigma$ consists of two states $1 \unlhd_{f} 2$, where $\Gamma^{1}=(), \Gamma^{2}=\left(\begin{array}{cccc}p & r & q & \omega \\ p & r & \omega & q\end{array}\right)$, and $f$ is constantly equal to 1 . To see this, consider the subcolumn $\delta=\left(\begin{array}{c}\sigma_{1} \\ \sigma_{2}\end{array}\right)$ of $f^{-1}((\sigma))$. Clearly, $2 \Vdash\left(\begin{array}{c}p \\ p\end{array}\right)$ and $2 \Vdash\left(\begin{array}{c}r \\ r\end{array}\right)$. But also $2 \Vdash\left(\begin{array}{c}r \rightarrow q \\ p \rightarrow q\end{array}\right)$. Indeed, the column in question has four subcolumns: $\left(\begin{array}{c}r \rightarrow q \\ p \rightarrow q\end{array}\right),\left(\begin{array}{cc}r \rightarrow q \\ \omega\end{array}\right),\left(\begin{array}{c}\omega \\ p \rightarrow q\end{array}\right),\left(\begin{array}{l}\omega \\ \omega\end{array}\right)$. The lhs of those are, respectively, $\left(\begin{array}{c}r \\ p\end{array}\right),\left(\begin{array}{c}r \\ \omega\end{array}\right),\left(\begin{array}{l}\omega \\ p\end{array}\right),\left(\begin{array}{c}\omega \\ \omega\end{array}\right)$, and all but the first one are forced in state 2 . 
The rhs of the forced ones $\left(\begin{array}{c}q \\ \omega\end{array}\right),\left(\begin{array}{c}\omega \\ q\end{array}\right),\left(\begin{array}{c}\omega \\ \omega\end{array}\right)$ are forced as well. Similarly, $2 \Vdash\left(\begin{array}{l}p \rightarrow q \\ r \rightarrow q\end{array}\right)$, and we conclude that $\operatorname{lhs}(\delta), \operatorname{lhs}(\operatorname{rhs}(\delta))$, lhs $(\operatorname{rhs}(\operatorname{rhs}(\delta)))$, and $\operatorname{lhs}(\operatorname{rhs}(\operatorname{rhs}(\operatorname{rhs}(\delta))))$, are forced in state 2 while $\operatorname{rhs}(\operatorname{rhs}(\operatorname{rhs}(\operatorname{rhs}(\delta))))$ is not.

Example 19. It may be instructive to observe that the $(\sigma)$ of Example 18 is forced in state $\mathbf{1}$ in the model obtained from the one above by assuming $\Gamma^{2}=\left(\begin{array}{ll}p & r \\ p & r\end{array}\right)$.

Since $1 \unlhd_{\text {id }} 1$ and $1 \unlhd_{f} 2$, we should check lhs and rhs of all subcolumns of $(\sigma)$ and of $f^{-1}((\sigma))$. The column $(\sigma)$ has four subcolumns, namely $(\sigma),\left(\sigma_{1}\right),\left(\sigma_{2}\right)$, and $(\omega)$. Only lhs $(\omega)=(\omega)$ is forced in $\mathbf{1}$, and since $\operatorname{rhs}(\omega)=(\omega)$ is forced as well, we conclude that the forcing condition is satisfied by state 1 and all subcolumns of $(\sigma)$.

Now consider state 2 and the 16 subcolumns of $f^{-1}((\sigma))=\left(\begin{array}{l}\sigma \\ \sigma\end{array}\right)$. Each of them has the form $\left(\begin{array}{l}\alpha \\ \beta\end{array}\right)$, where $\alpha, \beta \in\left\{\sigma, \sigma_{1}, \sigma_{2}, \omega\right\}$. Hence, the lhs of these columns are $\left(\begin{array}{c}\alpha^{\prime} \\ \beta^{\prime}\end{array}\right)$ with $\alpha^{\prime}, \beta^{\prime} \in\{p \cap p, p, \omega\}$, and each of them is forced in state 2 .

Now we show that rhs of all 16 is forced too. We look at the lhs of them, each one of the form $\left(\begin{array}{c}\alpha^{\prime \prime} \\ \beta^{\prime \prime}\end{array}\right)$ with $\alpha^{\prime \prime}, \beta^{\prime \prime} \in\{(r \rightarrow q) \cap(p \rightarrow q), r \rightarrow q, p \rightarrow q, \omega\}$. Then lhs $\left(\begin{array}{c}\alpha^{\prime \prime} \\ \beta^{\prime \prime}\end{array}\right)=\left(\begin{array}{l}\alpha^{\prime \prime \prime} \\ \beta^{\prime \prime \prime}\end{array}\right)$ with $\alpha^{\prime \prime \prime}, \beta^{\prime \prime \prime} \in\{r \cap p, r, p, \omega\}$, and there are 16 of them. Of those 16 , only 9 are forced at state 2 , namely $\left(\begin{array}{c}p \\ p\end{array}\right),\left(\begin{array}{c}r \\ r\end{array}\right)$, and 7 of the form $\left(\begin{array}{c}\alpha^{\prime \prime \prime} \\ \omega\end{array}\right)$ or $\left(\begin{array}{c}\omega \\ \beta^{\prime \prime \prime}\end{array}\right)$. Among the corresponding rhs, all but $\left(\begin{array}{c}\omega \\ \omega\end{array}\right)$ have at least one occurrence of $q$ and are not forced at 2 , hence all but one of our $\left(\begin{array}{c}\alpha^{\prime \prime} \\ \beta^{\prime \prime}\end{array}\right)$ are not forced at 2 . In other words, the lhs of 15 subcolumns $\left(\begin{array}{c}\alpha \\ \beta\end{array}\right)$ of $f^{-1}((\sigma))$ are not forced in state 2 . The one that is forced is $\left(\begin{array}{c}\omega \\ \omega\end{array}\right)$ and the corresponding rhs is also trivially forced. This concludes our verification.

Example 20. Let $\sigma$ be the following formula:

$(((p \rightarrow q) \rightarrow r) \rightarrow((q \rightarrow p) \rightarrow r) \rightarrow r \rightarrow r) \cap((\omega \rightarrow r) \rightarrow(\omega \rightarrow r) \rightarrow \omega \rightarrow r)$.

This is an intersection of two trivially inhabited types not containing occurrences of $\cap$ (morally, intuitionistic theorems). Yet it has a Kripke countermodel with $C=\{1,2,3,4\}$. Define $\Gamma^{1}=()$, $\Gamma^{2}=\left(\begin{array}{ll}r & \omega \\ \omega & r\end{array}\right), \Gamma^{3}=\left(\begin{array}{cccc}r & \omega & p & r \\ \omega & r & \omega & r\end{array}\right), \Gamma^{4}=\left(\begin{array}{cccc}r & \omega & q & r \\ \omega & r & \omega & r\end{array}\right)$. The order of states is $1 \leq 2 \leq 3,4$ with incomparable final states 3,4 . Of course, $\mathcal{H}(1 \leq C)$, for $C \neq 1$ is the constant function 1 , whereas $\mathcal{H}(C \leq D)$ is identity otherwise. We leave the details to the reader.

Example 21. Another nice exercise is the formula $\sigma=\sigma_{1} \cap \sigma_{2}$, where

$$
\begin{aligned}
& \sigma_{1}=((r \rightarrow p) \rightarrow p) \rightarrow(p \rightarrow q) \rightarrow((q \rightarrow r) \rightarrow r) \rightarrow(r \rightarrow q) \rightarrow r \rightarrow \omega \rightarrow q, \\
& \sigma_{2}=((r \rightarrow p) \rightarrow p) \rightarrow(p \rightarrow q) \rightarrow((q \rightarrow r) \rightarrow r) \rightarrow(r \rightarrow q) \rightarrow \omega \rightarrow r \rightarrow q .
\end{aligned}
$$

A countermodel for $(\sigma)$ uses three states $1 \leq 2 \leq 3$, where $\Gamma^{1}=(), \Gamma^{2}=\left(\begin{array}{cccc}r & \omega & q & \omega \\ \omega & r & \omega & q\end{array}\right)$, and $\Gamma^{3}=\left(\begin{array}{ccccc}r & \omega & q & \omega & q \\ \omega & r & \omega & q & q\end{array}\right)$. The function $\mathcal{H}$ is like in Example 20.

Observe that the model cannot be reduced to two states, as the column $\left(\begin{array}{c}\sigma_{1} \\ \sigma_{2}\end{array}\right)$ is forced in any final state $C$ of two columns. ${ }^{1}$ Suppose otherwise, then the columns $\left(\begin{array}{c}r \rightarrow q \\ r \rightarrow q\end{array}\right),\left(\begin{array}{c}r \\ \omega\end{array}\right),\left(\begin{array}{c}\omega \\ r\end{array}\right)$ must be forced in $C$, as otherwise the whole formula is trivially forced. Hence, $\left(\begin{array}{c}q \\ \omega\end{array}\right)$ and $\left(\begin{array}{c}\omega \\ q\end{array}\right)$ are forced as well, although $\left(\begin{array}{c}r \\ r\end{array}\right)$ is forbidden. Thus, $C \nVdash\left(\begin{array}{c}q \rightarrow r \\ q \rightarrow r\end{array}\right)$. But $C$ forces the three proper subcolumns of it: $\left(\begin{array}{c}q \rightarrow r \\ \omega\end{array}\right)$, $\left(\begin{array}{c}\omega \\ q \rightarrow r\end{array}\right)$, and $\left(\begin{array}{c}\omega \\ \omega\end{array}\right)$. So the full column $\left(\begin{array}{c}q \rightarrow r \\ q \rightarrow r\end{array}\right)$ is the only possible witness, but that can only happen when $C \Vdash\left(\begin{array}{c}q \\ q\end{array}\right)$, contradiction.

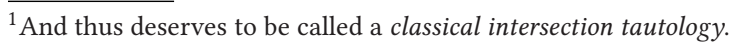




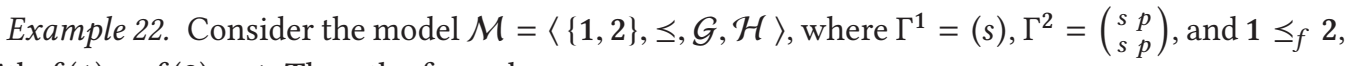
with $f(1)=f(2)=1$. Then the formula

$$
s \rightarrow(((s \rightarrow p) \rightarrow(r \rightarrow q) \rightarrow q) \cap((s \rightarrow p) \rightarrow(q \rightarrow r) \rightarrow r) \rightarrow p) \rightarrow p
$$

is not forced in state $\mathbf{1}$ of $\mathcal{M}$. Indeed, let $\gamma$ be the column consisting of the single entry $((s \rightarrow p) \rightarrow(r \rightarrow q) \rightarrow q) \cap((s \rightarrow p) \rightarrow(q \rightarrow r) \rightarrow r) \rightarrow p$. Then we have $1 \Vdash s, 1 \Vdash \gamma$, and $1 \nVdash p$. To see that $1 \Vdash \gamma$, observe that the only subcolumn $v$ of $f^{-1}(\gamma)$ such that $1 \Vdash \operatorname{lhs}(v)$ is $\omega$. For example, $1 \nVdash \operatorname{lhs}\left(f^{-1}(\gamma)\right)$, because $2 \Vdash\left(\begin{array}{c}s \rightarrow p \\ s \rightarrow p\end{array}\right)$ and $2 \Vdash\left(\begin{array}{c}r \rightarrow q \\ q \rightarrow r\end{array}\right)$, but $2 \nVdash\left(\begin{array}{c}q \\ r\end{array}\right)$.

We now show that forcing in monotone models respects the subtype relation $\leq$ (Lemma 24). First we prove that there must be a functional witness to every failure.

Lemma 23. If $C \Vdash \gamma, \gamma \leq \delta$, and $C \nVdash \delta$, then there exist functional $\gamma^{\prime}$, $\delta^{\prime}$ such that $C \Vdash \gamma^{\prime}, \gamma^{\prime} \leq \delta^{\prime}$, $C \nVdash \delta^{\prime}$, and $\min \left\{\operatorname{width}\left(\gamma^{\prime}\right)\right.$, width $\left.\left(\delta^{\prime}\right)\right\} \leq \min \{\operatorname{width}(\gamma)$, width $(\delta)\}$.

Proof. Necessarily, $\delta$ is functional. In case $\gamma$ is functional, taking $\gamma^{\prime}=\gamma$ and $\delta^{\prime}=\delta$ shows the claim. Next we consider the case in which $\gamma$ is atomic. There exists a functional $v \subseteq \gamma$ such that $v \leq \delta$. In case $C \Vdash v$, taking $\gamma^{\prime}=v$ and $\delta^{\prime}=\delta$ shows the claim. It remains to inspect the case in which $C \nVdash v$. Since $C \Vdash \gamma$ and $\gamma$ is atomic, there exists $\rho \in \Gamma^{C}$ such that $\rho \leq \gamma$. Therefore, there exists a functional $\mu \subseteq \rho$ such that $C \Vdash \mu$ and $\mu \leq \nu \leq \delta$. In case $\operatorname{width}(\gamma) \leq \operatorname{width}(\delta)$, taking $\gamma^{\prime}=\mu$ and $\delta^{\prime}=v$ shows the claim. In case width $(\delta) \leq \operatorname{width}(\gamma)$, taking $\gamma^{\prime}=\mu$ and $\delta^{\prime}=\delta$ shows the claim.

Lemma 24 (Monotonicity II). If $C \Vdash \gamma$ and $\gamma \leq \delta$, then $C \Vdash \delta$.

Proof. Assume (for contradiction) that $C \Vdash \gamma, \gamma \leq \delta$, and $C \nVdash \delta$, where $\gamma$ and $\delta$ are such that $\min \{\operatorname{width}(\gamma), \operatorname{width}(\delta)\}$ is minimal. By Lemma 23, we can assume that $\gamma$ and $\delta$ are functional.

Since $C \nVdash \delta$, there exist $C \unlhd_{f} D$ and $v \subseteq f^{-1}(\delta)$ such that $D \Vdash \operatorname{lhs}(v)$ and $D \nVdash \operatorname{rhs}(v)$. By Lemma 5, there exists $\mu \subseteq f^{-1}(\gamma)$ such that $\operatorname{lhs}(v) \leq \operatorname{lhs}(\mu)$ and $\operatorname{rhs}(\mu) \leq \operatorname{rhs}(v)$. Since $C \Vdash \gamma$, we have $D \nVdash \operatorname{lhs}(\mu)$ or $D \Vdash \operatorname{rhs}(\mu)$.

Observe that $\min \{\operatorname{width}(\operatorname{lhs}(v))$, width $(\operatorname{lhs}(\mu))\}<\min \{\operatorname{width}(\gamma), \operatorname{width}(\delta)\}$. Therefore, if we have $D \nVdash \operatorname{lhs}(\mu)$, then $\operatorname{lhs}(v) \leq \operatorname{lhs}(\mu)$ contradicts the minimality of $\min \{\operatorname{width}(\gamma)$, width $(\delta)\}$.

In case $D \Vdash \operatorname{rhs}(\mu)$, a similar contradiction is obtained from the fact $\operatorname{rhs}(\mu) \leq \operatorname{rhs}(v)$, since $\min \{\operatorname{width}(\operatorname{rhs}(\mu))$, width $(\operatorname{rhs}(v))\}<\min \{\operatorname{width}(\gamma), \operatorname{width}(\delta)\}$ as well.

We write $C \Vdash \Gamma$ if $C$ forces all columns in $\Gamma$. The notation $\Gamma \Vdash \gamma$ means, as is usual, the following: For every monotone model $\mathcal{M}$ and every state $C$, if $C \Vdash \Gamma$, then $C \Vdash \gamma$.

LEMMA 25 (Soundness). If $\Gamma \vdash \gamma$, then $\Gamma \Vdash \gamma$.

Proof. We need a stronger induction hypothesis. If $d \subseteq\left\{1, \ldots, m_{1}\right\} \times\left\{1, \ldots, m_{2}\right\}$ is any relation (called distribution), and $\gamma=\left(\alpha_{1}, \ldots, \alpha_{m_{1}}\right)$ is a column of height $m_{1}$, then we define a column $d(\gamma)=\left(\sigma_{1}, \ldots, \sigma_{m_{2}}\right)$, where $\sigma_{\ell}=\bigcap\left\{\alpha_{i} \mid\langle i, \ell\rangle \in d\right\}$. The matrix $d(\Gamma)$ is defined by columnwise application of $d$. We write $\Gamma \Perp \gamma$ when the following condition holds:

For every monotone model $\mathcal{M}$, every state $C$, and every distribution $d$, if $C \Vdash d(\Gamma)$, then $C \Vdash d(\gamma)$.

We now show by structural induction with respect to proofs that $\Gamma \vdash \gamma$ implies $\Gamma \Perp \vdash$. We consider cases depending on the last rule used. Cases (Cut) and $(\Omega)$ are immediate from the definition. Case (A) follows from Lemma 24, because $\Gamma \leq \gamma$ implies $d(\Gamma) \leq d(\gamma)$, for every distribution $d$.

(L) Assume $\Gamma, \operatorname{rhs}(\gamma) \Vdash \delta$ and $\Gamma \Vdash \operatorname{lhs}(\gamma)$, then suppose that $C \Vdash d(\Gamma)$. Since $\Gamma \Vdash \operatorname{lhs}(\gamma)$, we have $C \Vdash d(\operatorname{lhs}(\gamma))$, whence $C \Vdash d(\operatorname{rhs}(\gamma))$ by definition, because $C \unlhd_{\mathrm{id}} C$, the column $\gamma$ is a functional subcolumn of $\Gamma$, and lhs $(d(\gamma))=d(\operatorname{lhs}(\gamma))$. Then $\Gamma, \operatorname{rhs}(\gamma) \Vdash \delta$ yields $C \Vdash d(\delta)$. 
(R) Assume that $f:\left\{1, \ldots, m_{1}\right\} \rightarrow\{1, \ldots, m\}$ so that $\Gamma$ and $f^{-1}(\Gamma)$ have $m$ and $m_{1}$ rows, respectively. Let $\gamma=\left(\alpha_{1}, \ldots, \alpha_{m_{1}}\right)$ and $\delta=\left(\beta_{1}, \ldots, \beta_{m_{1}}\right)$. The $i$-th coordinate of the column $f(\gamma \Rightarrow \delta)$ is $\bigcap\left\{\alpha_{k} \rightarrow \beta_{k} \mid k \leq m_{1}\right.$ and $\left.f(k)=i\right\}$. The induction hypothesis states that $f^{-1}(\Gamma), \gamma \| t \delta$ and we want to prove $\Gamma \Vdash f(\gamma \Rightarrow \delta)$. Consider a distribution $d \subseteq\{1, \ldots, m\} \times\left\{1, \ldots, m_{2}\right\}$ and assume that $C \Vdash d(\Gamma)$. Let $C \leq_{g} D$, for some surjection $g:\left\{1, \ldots, m_{3}\right\} \rightarrow\left\{1, \ldots, m_{2}\right\}$. Take a subcolumn $v$ of $g^{-1}(d(f(\gamma \Rightarrow \delta)))$ such that $D \Vdash \operatorname{lhs}(v)$. We show that $D \Vdash \operatorname{rhs}(v)$.

The column $d(f(\gamma \Rightarrow \delta))$ is of height $m_{2}$ and its $\ell$-th coordinate is $\tau_{\ell}=\bigcap\left\{\alpha_{k} \rightarrow \beta_{k} \mid k \leq m_{1}\right.$ and $\left.\langle f(k), \ell\rangle \in d\right\}$.

The column $g^{-1}\left(d(f(\gamma \Rightarrow \delta))\right.$ is of height $m_{3}$ and its $j$-th coordinate is $\tau_{g(j)}$.

Let $K_{j}$ be sets such that the $j$-th coordinate of $v$ is

$$
\rho_{j}=\bigcap\left\{\alpha_{k} \rightarrow \beta_{k} \mid k \leq m_{1} \text { and }\langle f(k), g(j)\rangle \in d \text { and } k \in K_{j}\right\} .
$$

We see that $\operatorname{lhs}(v)$ is obtained by distribution of $\gamma$; indeed, we have $\operatorname{lhs}(v)=e(\gamma)$, where the distribution $e \subseteq\left\{1, \ldots, m_{1}\right\} \times\left\{1, \ldots, m_{3}\right\}$ is such that, for $k \leq m_{1}, j \leq m_{3}$ :

$$
\langle k, j\rangle \in e \text {, if and only if }\langle f(k), g(j)\rangle \in d \text { and } k \in K_{j} .
$$

It follows that $D \Vdash e(\gamma)$. Now we show that $D \Vdash e\left(f^{-1}(\Gamma)\right)$. We know that $C \Vdash d(\Gamma)$, and therefore $D \Vdash g^{-1}(d(\Gamma))$, by Lemma 13. Assume that $\Gamma=\left[\tau_{i x}\right]_{x=1 \ldots w}^{i=1 \ldots w}$. The type at position $(j, x)$ in $g^{-1}(d(\Gamma))$ has the form $\mu_{j x}=\bigcap\left\{\tau_{i x} \mid\langle i, g(j)\rangle \in d\right\}$, and we can write $\mu_{j x}=\bigcap\left\{\tau_{f(k) x} \mid\langle f(k), g(j)\rangle \in d\right\}$. From this, we conclude that $g^{-1}(d(\Gamma)) \leq e\left(f^{-1}(\Gamma)\right)$, componentwise, whence $D \Vdash e\left(f^{-1}(\Gamma)\right)$, by Lemma 24. By the induction hypothesis, $D \Vdash e(\delta)$. The claim follows due to $e(\delta)=\operatorname{rhs}(v)$.

\section{GAME PLAYING}

Our game corresponds to cut-free sequent calculus. There are two players. One is $\exists$ ros, the prover, and the other one is $\forall$ phrodite, the refuter. A position is a judgment $\mathcal{P}=(\Gamma \vdash \delta)$. Such a position is final when either $\delta=\omega$ or $\Gamma \leq \delta$. We use the notation $\delta_{\mathcal{P}}=\delta$. The game begins in an arbitrary initial position.

Every game turn (in a non-final position) is initiated by $\exists$ ros, who chooses a column: either the target column $\delta$ or a subcolumn of $\Gamma$. The chosen column must be functional. Every such choice corresponds to rule $(\mathrm{L})$ or $(\mathrm{R})$ seen as a proof tactic. Then the next position is determined by $\forall$ phrodite, who has as many choices as there are premises of the rule in question. If no functional column is available, the current position is repeated (the play enters a loop).

CASE L: An ᄏros' choice of a subcolumn $\gamma$ of $\Gamma$ corresponds to rule (L). Then $\forall$ phrodite chooses between two possible successor positions: either the position $(\Gamma, \operatorname{rhs}(\gamma) \vdash \delta)$ or the position $(\Gamma \vdash \operatorname{lhs}(\gamma))$.

CASE R: A choice of a target column $\delta=\left(\bigcap_{k \in I_{1}}\left(\alpha_{1 k} \rightarrow \beta_{1 k}\right), \ldots, \bigcap_{k \in I_{m}}\left(\alpha_{m k} \rightarrow \beta_{m k}\right)\right)$ corresponds to rule (R). Assume without loss of generality that the sets $I_{1}, \ldots, I_{m}$ are disjoint, and $\bigcup_{j} I_{j}=\left\{1, \ldots, m^{\prime}\right\}$, for some $m^{\prime} \geq m$. Define $f:\left\{1, \ldots, m^{\prime}\right\} \rightarrow\{1, \ldots, m\}$ so that

$$
\left.f(k)=i \text {, for } k \in I_{i} \text { (that is, } f^{-1}(\{i\})=I_{i}\right) \text {. }
$$

Also define $\gamma=\left(\tau_{1}, \ldots, \tau_{m^{\prime}}\right)$ and $\zeta=\left(\sigma_{1}, \ldots, \sigma_{m^{\prime}}\right)$ so that $\tau_{k}=\alpha_{f(k) k}$ and $\sigma_{k}=\beta_{f(k) k}$, for $k=1 \ldots m^{\prime}$. We have $\delta=f(\gamma \Rightarrow \zeta)$, and $\forall$ phrodite must set $\left(f^{-1}(\Gamma), \gamma+\zeta\right)$ as the next position.

Consider a game turn leading from a position $\mathcal{P}=(\Gamma \vdash \delta)$ to a position $\mathcal{P}^{\prime}=\left(\Gamma^{\prime} \vdash \delta^{\prime}\right)$. Let $\Gamma$ and $\Gamma^{\prime}$ have $m$ and $m^{\prime}$ rows, respectively. The principal collapse from $\mathcal{P}^{\prime}$ to $\mathcal{P}$ is a surjection $f:\left\{1, \ldots, m^{\prime}\right\} \rightarrow\{1, \ldots, m\}$ such that $f=\mathrm{id}$ in case (L), and $f$ is defined by $\left(^{*}\right)$ in case (R). Then we have $\Gamma \sqsubseteq_{f} \Gamma^{\prime}$.

A play (a sequence of turns) is won by $\exists$ ros, when it ends in a final position. Otherwise, the play is infinite, in which case $\forall$ phrodite wins.

A winning strategy of $\exists$ ros in position $\mathcal{P}_{0}$ is a finite tree $\mathcal{T}$ labeled by positions, such that the root is labeled $\mathcal{P}_{0}$, and one of the following holds: 
- Position $\mathcal{P}_{0}$ is final and $\mathcal{T}$ consists only of the root.

- There is an ᄏros' move leading to one or two successor positions available for $\forall$ phrodite, and the immediate subtrees of $\mathcal{T}$ are $\exists$ ros' strategies in these positions.

Dually, we define a strategy of $\forall$ phrodite in position $\mathcal{P}_{0}$ as an infinite tree $\mathcal{S}$ labeled by non-final positions such that the root is labeled by $\mathcal{P}_{0}$ and

- For every node in $\mathcal{S}$, labeled $\mathcal{P}$, and every $\exists$ ros' move possible in $\mathcal{P}$, there is in $\mathcal{S}$ a successor labeled by a position $\mathcal{P}^{\prime}$ obtained by that $\exists$ ros' move followed by a response of $\forall$ phrodite (i.e., by a single game turn).

The following should now be obvious.

Proposition 26. Let $\Gamma \vdash \gamma$ be an initial position in a game. Then

(1) The game is determined: either $\exists$ ros or $\forall$ phrodite has a winning strategy.

(2) A winning strategy of $\exists$ ros defines a cut-free proof of $\Gamma \vdash \gamma$.

Proof. (1) Assume that $\exists$ ros does not have a strategy, in position $\mathcal{P}$. Then $\mathcal{P}$ is not final and every move $\exists$ ros can make in position $\mathcal{P}$ can be completed by $\forall$ phrodite's response leading to another position where $\exists$ ros still has no strategy. In this way, one can build an infinite strategy of $\forall$ phrodite. (2) Follows directly from Lemma 11, since ヨros' strategy is nothing else than a cut-free proof in disguise.

Example 27. Let $\alpha=((p \rightarrow q) \rightarrow(p \rightarrow p) \rightarrow r) \cap((p \rightarrow p) \rightarrow(p \rightarrow q) \rightarrow r) \rightarrow r$, and $\beta=(q \rightarrow p \rightarrow r) \cap(p \rightarrow q \rightarrow r)$. In a game commencing in position $\vdash(\alpha \rightarrow \beta \rightarrow p \rightarrow r)$, $\exists$ ros has a winning strategy. He begins with three (R) moves leading to position $(\alpha \beta p) \vdash(r)$ and then he plays move (L) with $\alpha$. In response to this, $\forall$ phrodite must set as the new target $((p \rightarrow q) \rightarrow(p \rightarrow p) \rightarrow r) \cap((p \rightarrow p) \rightarrow(p \rightarrow q) \rightarrow r)$, as she does not want to find herself in a final position. rros responds with two more (R) moves and this puts the play in position $\left(\begin{array}{lllll}\alpha & \beta & p & p \rightarrow q & p \rightarrow p \\ \alpha & \beta & p & p \rightarrow p & p \rightarrow q\end{array}\right) \vdash\left(\begin{array}{l}r \\ r\end{array}\right)$. Now $\exists$ ros plays move (L) using the subcolumn $\left(\begin{array}{l}q \rightarrow p \rightarrow r \\ p \rightarrow q \rightarrow r\end{array}\right)$ of column $\left(\begin{array}{l}\beta \\ \beta\end{array}\right)$. Suppose that $\forall$ phrodite in response changes the target column to $\left(\begin{array}{l}q \\ p\end{array}\right)$. Then $\exists$ ros plays (L) using $\left(\begin{array}{c}p \rightarrow q \\ p \rightarrow p\end{array}\right)$. Every $\forall$ phrodite's move now leads to a final position.

The other case is that $\forall$ phrodite allows $\exists$ ros to use a new column at the left and the position becomes $\left(\begin{array}{cccccc}\alpha & \beta & p & p \rightarrow q & p \rightarrow p & p \rightarrow r \\ \alpha & \beta & p & p \rightarrow p & p \rightarrow q & q \rightarrow r\end{array}\right) \vdash\left(\begin{array}{l}r \\ r\end{array}\right)$. Then $\exists$ ros uses just this new column $\left(\begin{array}{c}p \rightarrow r \\ q \rightarrow r\end{array}\right)$ in his next (L) move. $\forall$ phrodite must now set $\left(\begin{array}{l}p \\ q\end{array}\right)$ as the new target, and she loses in the next step because $\exists$ Jros can refer to the assumption $\left(\begin{array}{c}p \rightarrow p \\ p \rightarrow q\end{array}\right)$.

The preceding strategy corresponds to a normal inhabitant of type $\alpha \rightarrow \beta \rightarrow p \rightarrow r$, namely $\lambda x y z \cdot x(\lambda u v \cdot y(u z)(v z))$.

Example 28. Let $\sigma$ be the formula of Example 18. If the initial position is $\vdash(\sigma)$, then the first two moves of $\exists$ ros must be (R) moves leading to position $\left(\begin{array}{cc}p & r \rightarrow q \\ p & p \rightarrow q\end{array}\right) \vdash\left(\begin{array}{l}r \rightarrow(p \rightarrow q) \rightarrow q \\ r \rightarrow(r \rightarrow q) \rightarrow q\end{array}\right)$. Now $\exists$ ros can play (R) two more times, but once functional columns begin to appear on the left, he may also play (L) moves. If he chooses the full column $\left(\begin{array}{c}r \rightarrow q \\ p \rightarrow q\end{array}\right)$, then $\forall$ phrodite can respond by changing the target to $\left(\begin{array}{l}r \\ p\end{array}\right)$. If a proper subcolumn $\left(\begin{array}{c}r \rightarrow q \\ \omega\end{array}\right),\left(\begin{array}{c}\omega \\ p \rightarrow q\end{array}\right)$, or $\left(\begin{array}{c}\omega \\ \omega\end{array}\right)$, is selected, $\forall$ phrodite will add a new column at the left, respectively $\left(\begin{array}{c}q \\ \omega\end{array}\right),\left(\begin{array}{c}\omega \\ q\end{array}\right)$, or $\left(\begin{array}{c}\omega \\ \omega\end{array}\right)$, the latter of course just ritually. In an analogous way, $\forall$ phrodite would react to $\exists$ ros' attempts to play (L) using subcolumns of $\left(\begin{array}{c}p \rightarrow q \\ r \rightarrow q\end{array}\right)$.

These moves can happen in various order, leading always to positions where the left-hand side consists of subcolumns of the matrix $\left(\begin{array}{cccccc}p & r \rightarrow q & r & p \rightarrow q & q & \omega \\ p & p \rightarrow q & r & r \rightarrow q & \omega & q\end{array}\right)$, and the right-hand side is either $\left(\begin{array}{l}q \\ q\end{array}\right),\left(\begin{array}{l}r \\ p\end{array}\right)$, or $\left(\begin{array}{l}p \\ r\end{array}\right)$. In either case, $\exists$ ros does not have access to an appropriate atomic column and he cannot win. 
Example 29. Let $\sigma$ be as in Example 20. In the game commencing at $\vdash(\sigma), \forall$ phrodite must adjust her choices to $\exists$ ros' behavior. Consider a play beginning with three (R) moves followed by $\exists$ ros' choice of the column $\delta_{1}=\left(\begin{array}{c}p \rightarrow q) \rightarrow r \\ \omega \rightarrow r\end{array}\right)$. Then $\forall$ phrodite must respond by setting new target as $\left(\begin{array}{c}p \rightarrow q \\ \omega\end{array}\right)$. Jros may select the target column, and this adds a new column $\left(\begin{array}{l}p \\ \omega\end{array}\right)$ at the left and changes the target again into $\left(\begin{array}{c}q \\ \omega\end{array}\right)$. Dually, if $\exists$ ros first plays (L) with $\delta_{2}=\left(\begin{array}{c}(q \rightarrow p) \rightarrow r \\ \omega \rightarrow r\end{array}\right)$, instead of $\delta_{1}$, and then $(\mathrm{R})$, then $\forall$ phrodite's response eventually adds $\left(\begin{array}{c}q \\ \omega\end{array}\right)$ toward a new target $\left(\begin{array}{l}p \\ \omega\end{array}\right)$. But if $\exists$ ros executes both these scenarios one after the other, $\forall$ phrodite must act differently: she cannot approve a target that already occurs at the left. Instead she would add the column $\left(\begin{array}{l}r \\ r\end{array}\right)$ to assumptions, being confident that it will never again occur as target.

We now show that a winning strategy of $\forall$ phrodite (infinite refutation) yields a countermodel. The proof is an adaptation of the one in the work of Urzyczyn [18, Section 2].

From now on, we fix a winning strategy $\mathcal{S}$ at position $\mathcal{P}_{0}$. An edge in $\mathcal{S}$ (a game turn) leading from a position $\mathcal{P}=(\Gamma \vdash \delta)$ to a position $\mathcal{P}^{\prime}=(\Gamma, \gamma \vdash \delta)$ with the same target column is called static. If the added $\gamma$ is already a column of $\Gamma$, then we call the edge idle. A position $\mathcal{P}$ in $\mathcal{S}$ is saturated if every static turn from $\mathcal{P}$ in $\mathcal{S}$ is idle.

Lemma 30. Every $\mathcal{P}=(\Gamma \vdash \delta) \in \mathcal{S}$ has a saturated descendant $\mathcal{P}^{\prime}=\left(\Gamma^{\prime} \vdash \delta\right)$ in $\mathcal{S}$ such that $\Gamma \sqsubseteq_{\mathrm{id}} \Gamma^{\prime}$ and the target column $\delta$ remains the same.

Proof. Every path in $\mathcal{S}$ originating in $\mathcal{P}$ represents a play segment where $\forall$ phrodite follows her strategy against some sequence of $\exists$ ros' moves. There are plays (branches in $\mathcal{S}$ ) determined by arbitrary behaviors of $\exists$ ros. In particular, there are branches consisting solely of static turns. Since the number of possibly introduced columns is finite, every such branch must eventually reach a saturated position.

Example 31. Let $\mathcal{S}$ be the winning strategy of $\forall$ phrodite as in Example 28. The initial position and the one immediately following it are trivially saturated because no static move can be played in these positions. But the next position $\left(\begin{array}{cc}p & r \rightarrow q \\ p & p \rightarrow q\end{array}\right) \vdash\left(\begin{array}{c}r \rightarrow(p \rightarrow q) \rightarrow q \\ r \rightarrow(r \rightarrow q) \rightarrow q\end{array}\right)$ is not saturated, because nonidle static moves are possible. For example, if $\exists$ ros selects the subcolumn $\left(\begin{array}{c}r \rightarrow q \\ \omega\end{array}\right)$ of $\left(\begin{array}{c}r \rightarrow q \\ p \rightarrow q\end{array}\right)$, then $\forall$ phrodite gives him a new assumption column $\left(\begin{array}{c}q \\ \omega\end{array}\right)$ that does not yet occur at the left. The position in question has a saturated descendant in $\mathcal{S}$, namely $\left(\begin{array}{ccccc}p & r \rightarrow q & q & \omega & \omega \\ p & p \rightarrow q & \omega & q & \omega\end{array}\right) \vdash\left(\begin{array}{l}r \rightarrow(p \rightarrow q) \rightarrow q \\ r \rightarrow(r \rightarrow q) \rightarrow q\end{array}\right)$, obtained when $\exists$ ros selects all proper subcolumns of $\left(\begin{array}{c}r \rightarrow q \\ p \rightarrow q\end{array}\right)$ in order. In summary, the saturated positions in this game are exactly those with matrices ()$,\left(\begin{array}{l}p \\ p\end{array}\right),\left(\begin{array}{llllll}p & r \rightarrow q & q & \omega & \omega \\ p & p \rightarrow q & \omega & q & \omega\end{array}\right),\left(\begin{array}{lllllll}p & r \rightarrow q & q & \omega & \omega & r \\ p & p \rightarrow q & \omega & q & \omega & r\end{array}\right)$, and $\left(\begin{array}{ccccccc}p & r \rightarrow q & q & \omega & \omega & r & p \rightarrow q \\ p & p \rightarrow q & \omega & q & \omega & r & r \rightarrow q\end{array}\right)$. Recall from Example 28 that if $\exists$ ros selects either of the two functional columns (not a subcolumn), then the resulting move in $\mathcal{S}$ is not static.

The model: Given a winning strategy $\mathcal{S}$ of $\forall$ phrodite, we define the Kripke model $\mathcal{M}_{\mathcal{S}}$ :

- States of $\mathcal{M}_{\mathcal{S}}$ are the saturated positions in $\mathcal{S}$.

- For $\mathcal{P}=(\Gamma \vdash \delta) \in \mathcal{M}_{\mathcal{S}}$, the matrix $\Gamma^{\mathcal{P}}$ consists of all atomic ${ }^{2}$ columns in $\Gamma$.

- The relation $\mathcal{P} \leq_{f} \mathcal{P}^{\prime}$ holds if $\mathcal{P}^{\prime}$ is a descendant of $\mathcal{P}$ in $\mathcal{S}$.

- If $\mathcal{P}^{\prime}$ is an immediate successor of $\mathcal{P}$ in $\mathcal{S}$, then $\mathcal{H}\left(\mathcal{P}, \mathcal{P}^{\prime}\right)$ is the principal collapse from $\mathcal{P}^{\prime}$ to $\mathcal{P}$. Otherwise, it is the appropriate composition.

Monotonicity of our model is an immediate consequence of the following.

${ }^{2}$ Note that typically $\Gamma^{\mathcal{P}}$ is not the same as $\Gamma$. 
Lemma 32. Let $\mathcal{P}=(\Gamma \vdash \delta)$ be a state of $\mathcal{M}_{\mathcal{S}}$. If $\Gamma \leq \gamma^{\prime}$, then $\mathcal{P} \Vdash \gamma^{\prime}$, and if $\delta^{\prime} \leq \delta$, then $\mathcal{P} \nVdash \delta^{\prime}$.

Proof. We prove the two parts by parallel induction with respect to the width of columns $\gamma^{\prime}$ and $\delta^{\prime}$. (It is a rough upper bound for the number of decompositions of a column into lhs and rhs.)

Let $\gamma \leq \gamma^{\prime}$, for some $\gamma \in \Gamma$. The case of an atomic $\gamma^{\prime}$ follows directly from the definition of forcing, so we may assume that $\gamma^{\prime}$ is functional. Take a state $\mathcal{R}=\left(\Gamma^{\prime} \vdash \delta^{\prime}\right)$ such that $\mathcal{P} \leq_{f} \mathcal{R}$, and let $v^{\prime}$ be a subcolumn of $f^{-1}\left(\gamma^{\prime}\right)$ such that $\mathcal{R} \Vdash \operatorname{lhs}\left(v^{\prime}\right)$. We prove that $\mathcal{R} \Vdash \operatorname{rhs}\left(v^{\prime}\right)$.

We have $f^{-1}(\gamma) \leq f^{-1}\left(\gamma^{\prime}\right)$. By Lemma 5 , there exists a subcolumn $v$ of $f^{-1}(\gamma)$ such that $\operatorname{lhs}\left(v^{\prime}\right) \leq \operatorname{lhs}(v)$ and $\operatorname{rhs}(v) \leq \operatorname{rhs}\left(v^{\prime}\right)$. By definition of our model $\mathcal{M}_{S}$, we have $\Gamma \sqsubseteq_{f} \Gamma^{\prime}$ (which is more than just $\Gamma^{\mathcal{P}} \sqsubseteq_{f} \Gamma^{\mathcal{R}}$ ). Hence, in position $\mathcal{R}$, ᄏros can play (L), selecting the subcolumn $v$. Then $\forall$ phrodite chooses the next position from the two options: $\left(\Gamma^{\prime}, \operatorname{rhs}(v) \vdash \delta^{\prime}\right)$ or $\left(\Gamma^{\prime} \vdash \operatorname{lhs}(v)\right)$. In other words, one of these positions is a successor of $\mathcal{R}$ in $\mathcal{S}$.

Suppose that $\forall$ phrodite chooses the second option. The obtained position extends to a saturated position $Q$ in $\mathcal{M}_{\mathcal{S}}$ (Lemma 30) with the same target column $\delta_{Q}=\operatorname{lhs}(v) \geq \operatorname{lhs}\left(v^{\prime}\right)$. By the induction hypothesis, we have $Q \nVdash \operatorname{lhs}\left(v^{\prime}\right)$. Since $Q \leftrightharpoons_{\text {id }} \mathcal{R}$, we also have $\mathcal{R} \nVdash \operatorname{lhs}\left(v^{\prime}\right)$ by Lemma 13 , and this is a contradiction.

Therefore, $\forall$ phrodite must choose the first option. Since $\mathcal{R}$ is saturated, static turns are idle, implying that the column $\operatorname{rhs}(v)$ is present in $\Gamma^{\prime}$. By the induction hypothesis, we obtain $\mathcal{R} \Vdash \operatorname{rhs}\left(v^{\prime}\right)$, showing the claim.

Now assume that $\delta^{\prime} \leq \delta$. If the column $\delta$ is atomic, then so is $\delta^{\prime}$. If $\mathcal{P} \Vdash \delta^{\prime}$, then $\Gamma^{\mathcal{P}} \leq \delta^{\prime} \leq \delta$, whence $\Gamma \leq \delta$. This cannot happen, because the position is not final.

So if $\mathcal{P} \Vdash \delta^{\prime}$, then $\delta$ must be functional. Therefore, $\exists$ ros can play move (R) at $\mathcal{P}$, resulting in position $f^{-1}(\Gamma), \gamma \vdash \zeta$ such that $\delta=f(\gamma \Rightarrow \zeta)$. This position extends to a saturated $\mathcal{R}=\left(\Gamma^{\prime} \vdash \zeta\right)$ in $\mathcal{M}_{\mathcal{S}}$ with $\gamma$ occurring in the matrix $\Gamma^{\prime}$. Since $\delta^{\prime} \leq f(\gamma \Rightarrow \zeta)$ there is, by Lemma 6, a functional subcolumn $v$ of $f^{-1}\left(\delta^{\prime}\right)$ such that $\gamma \leq \operatorname{lhs}(v)$ and $\operatorname{rhs}(v) \leq \zeta$. By the induction hypothesis, we have $\mathcal{R} \Vdash \operatorname{lhs}(v)$ and $\mathcal{R} \nVdash \operatorname{rhs}(v)$-a contradiction with $\mathcal{P} \Vdash \delta^{\prime}$.

Corollary 33. If $\forall$ phrodite has a winning strategy at position $\mathcal{P}_{0}=(\Gamma \vdash \delta)$, then $\Gamma \nVdash \delta$.

Proof. Let $\mathcal{S}$ be a winning strategy, and let $Q$ be a saturated descendant of $\mathcal{P}_{0}$ with $\delta_{Q}=\delta$. By Lemma 32, we have $Q \Vdash \Gamma$ and $Q \nVdash \delta$ in the model $\mathcal{M}_{\mathcal{S}}$.

Remark 34. Models $\mathcal{M}_{\mathcal{S}}$ are always infinite trees by definition. However, they often include a lot of repetitions and can sometimes be collapsed to finite models. Some of our models in Examples 20 through 22 can be seen as such collapses. Note that a model built from an actual strategy must reflect all possible behaviors of $\exists$ ros, including redundant and ineffective moves. For example, in the game induced by the formula of Example 22, ᄏros can unnecessarily play move (L). This results in positions with 4,8 , and more rows, to be represented by states of the infinite model.

However, the finite model property does not hold in general (Corollary 37$){ }^{3}$

Example 35 shows that even infinite models that contain no repetitions (but still have ineffective columns) may be collapsed.

Example 35. Consider the formula $\sigma=(q \rightarrow p) \cap(r \rightarrow p) \rightarrow p$ and the column $\delta=(\sigma \rightarrow p)$ of height 1 . Starting from position $(\varnothing \vdash \delta)$, $\exists$ ros will be forced by $\forall$ phrodite to repeatedly find himself in positions,

$$
\left((\sigma, \ldots, \sigma), \gamma_{1}, \ldots, \gamma_{n} \vdash(p, \ldots, p)\right)
$$

\footnotetext{
${ }^{3}$ However, it is challenging to construct a simple formula with a necessarily infinite countermodel.
} 
where $\gamma_{i}$ are distinct and consist of $q$ and $r$, for $i=1 \ldots n$. Since $\gamma_{1}, \ldots, \gamma_{n}$ are distinct and consist of atoms, the corresponding countermodel is not easily collapsible. Still, there exists a finite countermodel consisting of a single state 1 such that $\Gamma^{1}=(q)$. We have that $1 \nVdash(q \rightarrow p)$, and thus also $1 \nVdash(q \rightarrow p) \cap(r \rightarrow p)$ as $(q \rightarrow p)$ is a subcolumn. Therefore, $1 \Vdash \sigma$, and we obtain $1 \nVdash \delta$, because $1 \nVdash p$.

Theorem 36 (Soundness And Completeness). We have $\Gamma \vdash_{\mathrm{BCD}} \sigma$ if and only if $\Gamma \Vdash(\sigma)$. In particular, $\sigma$ is a theorem of intersection logic if and only if it is forced in every model.

Proof. Soundness holds by Lemmas 11 and 25. For completeness, assume $\Gamma \nvdash_{\mathrm{BCD}} \sigma$. By Lemma 11, we have $\Gamma \nvdash(\sigma)$, and thus $\forall$ phrodite has a winning strategy at position $(\Gamma \vdash(\sigma))$. By Corollary 33, we obtain $\Gamma \nVdash(\sigma)$.

By the preceding Theorem 36, to refute a formula semantically it suffices to provide a countermodel. As illustrated in Examples 17 through 22 and 35, this can be simpler than inspecting all possible derivations.

COROLLARY 37. Intersection logic does not have the finite model property: there exist unprovable formulas forced in every finite model.

Proof. Otherwise, the logic would be decidable and it is not the case [16].

Corollary 38 (Cut-elimination). The sequent calculus of Figure 1 has the cut-elimination property.

Proof. Assume that $\Gamma \vdash \gamma$ is provable. Then $\Gamma \Vdash \gamma$, by Lemma 25. Thus, by Corollary 33, $\forall$ phrodite has no winning strategy at position $(\Gamma \vdash \gamma)$, so $\exists$ ros must have one. By Proposition 26, there is a cut-free proof of $\Gamma \vdash \gamma$.

COROllary 39 (Normalization). If $\mathrm{\vdash}_{\mathrm{BC}} \sigma$, then $\sigma$ has an inhabitant in $\beta$-normal form.

Proof. By Corollary 38 and Lemma 11.

\section{UNIFORM RESTRICTION}

In this section, we restrict our attention to formulas that have uniform structure-that is, refine some simple type in the sense of Kohlhase and Pfenning [8].

Let $o$ be a fixed atom. For an intersection type $\sigma$ and a simple type $\tau$, we say that $\sigma$ refines $\tau$ if one of the following cases holds:

- $\sigma$ is an atom and $\tau=o$.

- $\sigma=\bigcap_{i \in I} \sigma_{i}$, where $\sigma_{i}$ refines $\tau$, for all $i \in I^{4}$

- $\sigma=\sigma_{1} \rightarrow \sigma_{2}$ and $\tau=\tau_{1} \rightarrow \tau_{2}$, where $\sigma_{1}$ refines $\tau_{1}$ and $\sigma_{2}$ refines $\tau_{2}$.

We say that $\sigma$ is uniform if $\sigma$ refines some simple type $\tau$. A column is uniform if the intersection of its coordinates is uniform. A matrix is uniform if every of its columns is uniform. We say that a Kripke model is uniform if for every of its states $C$ the matrix $\Gamma^{C}$ is uniform. Observe that if a column is uniform and atomic, then all of its coordinates are intersections of atoms.

Uniform models satisfy the monotonicity condition by definition. Indeed, in a uniform model, every $\Gamma^{C}$ consists of columns in which each coordinate is a (possibly empty) intersection of atoms.

${ }^{4}$ Specifically, the formula $\omega$ refines any simple type $\tau$. 
In the following, let us restrict attention to uniform models. In particular, for uniform $\Gamma$ and $\delta$, let us write $\Gamma \Vdash_{u} \delta$, meaning the following:

For every uniform model $\mathcal{M}$ and every state $C$, if $C \Vdash \Gamma$, then $C \Vdash \delta$.

Uniform forcing is sound and complete.

Lemma 40. Let $\Gamma$ and $\delta$ be uniform. We have $\Gamma \vdash \delta$ if and only if $\Gamma \Vdash_{u} \delta$.

Proof. $(\Rightarrow)$ Immediate from Theorem 36.

$(\Leftarrow)$ If $\Gamma \nvdash \delta$, then $\forall$ phrodite has a winning strategy $\mathcal{S}$ at position $\mathcal{P}_{0}=(\Gamma \vdash \delta)$. Since subcolumn selection, row copying and exchange, and decomposition into lhs/rhs preserve uniformity, the countermodel $\mathcal{M}_{\mathcal{S}}$ is uniform.

Theorem 41 (Uniform Soundness And Completeness). For uniform $\Gamma$ and $\sigma$, we have $\Gamma \vdash_{\mathrm{BCD}} \sigma$ if and only if $\Gamma \Vdash_{u}(\sigma)$.

Proof. Immediate consequence of Lemma 11 and Lemma 40.

\section{ACKNOWLEDGMENT}

We thank Luigi Liquori and Claude Stolze for helpful and accurate comments on the initial version of this article. We also feel indebted to the anonymous reviewers for their useful suggestions.

\section{REFERENCES}

[1] Henk Barendregt, Mario Coppo, and Mariangiola Dezani-Ciancaglini. 1983. A filter lambda model and the completeness of type assignment. fournal of Symbolic Logic 48, 4 (1983), 931-940. https://doi.org/10.2307/2273659

[2] Henk Barendregt, Wil Dekkers, and Richard Statman. 2013. Lambda Calculus with Types. Cambridge University Press.

[3] Mariangiola Dezani-Ciancaglini, Silvia Ghilezan, and Betti Venneri. 1997. The "relevance" of intersection and union types. Notre Dame Journal of Formal Logic 38, 2 (1997), 246-269. https://doi.org/10.1305/ndjfl/1039724889

[4] Melvin Fitting. 1969. Intuitionistic Logic, Model Theory and Forcing. North-Holland.

[5] Dov M. Gabbay. 1974. On 2nd order intuitionistic propositional calculus with full comprehension. Archiv für mathematische Logik und Grundlagenforschung 16 (1974), 177-186. https://doi.org/10.1007/bf02015377

[6] Dov M. Gabbay. 1981. Semantical Investigations in Heyting's Intuitionistic Logic. Synthese Library, Vol. 148. D. Reidel. https://doi.org/10.1007/978-94-017-2977-2

[7] Simona Kašterović and Silvia Ghilezan. 2020. Kripke-style semantics and completeness for full simply typed lambda calculus. Journal of Logic and Computation 30, 8 (2020), 1567-1608. https://doi.org/10.1093/logcom/exaa055

[8] Michael Kohlhase and Frank Pfenning. 1993. Unification in a lambda-calculus with intersection types. In Logic Programming, Proceedings of the 1993 International Symposium, Vancouver, British Columbia, Canada, October 26-29, 1993, Dale Miller (Ed.). MIT Press, Cambridge, MA, 488-505.

[9] Luigi Liquori and Claude Stolze. 2019. The Delta-calculus: Syntax and types. In 4th International Conference on Formal Structures for Computation and Deduction (FSCD 2019) (Leibniz International Proceedings in Informatics (LIPIcs)), Herman Geuvers (Ed.), Vol. 131. Schloss Dagstuhl-Leibniz-Zentrum fuer Informatik, Dagstuhl, Germany, Article 28, 20 pages. https://doi.org/10.4230/LIPIcs.FSCD.2019.28

[10] John C. Mitchell and Eugenio Moggi. 1991. Kripke-style models for typed lambda calculus. Annals of Pure and Applied Logic 51, 1-2 (1991), 99-124. https://doi.org/10.1016/0168-0072(91)90067-V

[11] Elaine Pimentel, Simona Ronchi Della Rocca, and Luca Roversi. 2012. Intersection types from a proof-theoretic perspective. Fundamenta Informaticae 121, 1-4 (2012), 253-274. https://doi.org/10.3233/FI-2012-778

[12] Garrel Pottinger. 1980. A type assignment for the strongly normalizable $\lambda$-terms. In To H.B. Curry: Essays on Combinatory Logic, Lambda Calculus and Formalism, J. P. Seldin and J. R. Hindley (Eds.). Academic Press, 561-577.

[13] Simona Ronchi Della Rocca and Luca Roversi. 2001. Intersection logic. In Computer Science Logic, 15th International Workshop, CSL 2001. (Lecture Notes in Computer Science), Laurent Fribourg (Ed.), Vol. 2142. Springer, 414-428. https: //doi.org/10.1007/3-540-44802-0_29

[14] Simona Ronchi Della Rocca, Alexis Saurin, Yiorgos Stavrinos, and Anastasia Veneti. 2010. Intersection logic in sequent calculus style. In Proceedings of the 5th Workshop on Intersection Types and Related Systems (ITRS'10), Vol. 45. 16-30. I https://doi.org/10.4204/EPTCS.45.2

[15] S. K. Sobolev. 1977. The intuitionistic propositional calculus with quantifiers. Mathematical Notes of the Academy of Sciences of the USSR 22, 1 (1977), 69-76. https://doi.org/10.1007/bf01147694 
[16] Paweł Urzyczyn. 1999. The emptiness problem for intersection types. fournal of Symbolic Logic 64, 3 (1999), $1195-1215$. https://doi.org/10.2307/2586625

[17] Paweł Urzyczyn. 2010. The logic of persistent intersection. Fundamenta Informaticae 103, 1-4 (2010), 303-322. https: //doi.org/10.3233/FI-2010-330

[18] Paweł Urzyczyn. 2016. Intuitionistic games: Determinacy, completeness, and normalization. Studia Logica 104, 5 (2016), 957-1001. https://doi.org/10.1007/s11225-016-9661-4

[19] Silvio Valentini and Matteo Viale. 2003. A binary modal logic for the intersection types of lambda-calculus. Information and Computation 185, 2 (2003), 211-232. https://doi.org/10.1016/S0890-5401(03)00089-0

[20] Betti Venneri. 1994. Intersection types as logical formulae. Journal of Logic and Computation 4, 2 (1994), $109-124$. https://doi.org/10.1093/logcom/4.2.109

[21] Hirofumi Yokouchi. 2002. Completeness of type assignment systems with intersection, union, and type quantifiers. Theoretical Computer Science 272, 1-2 (2002), 341-398. https://doi.org/10.1016/S0304-3975(00)00356-X

Received June 2020; revised November 2020; accepted March 2021 